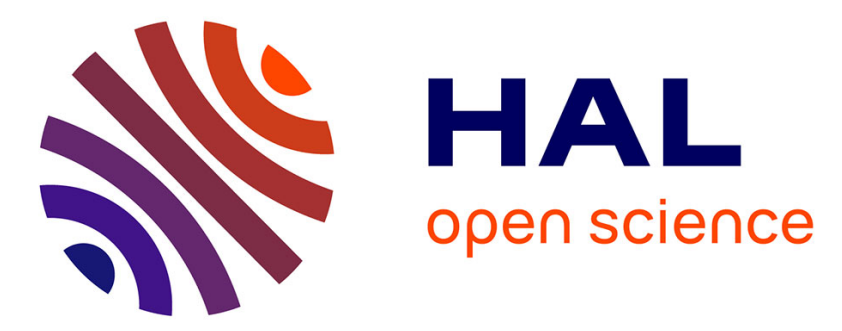

\title{
On Limitations of Using Silicon Photo-Multipliers for Underwater Wireless Optical Communications
}

Tasnim Hamza, Mohammad Ali Khalighi

\section{To cite this version:}

Tasnim Hamza, Mohammad Ali Khalighi. On Limitations of Using Silicon Photo-Multipliers for Underwater Wireless Optical Communications. IEEE WACOWC Conference, Apr 2019, Tehran, Iran. 10.1109/WACOWC.2019.8770202 . hal-02423695

\section{HAL Id: hal-02423695 \\ https://hal.science/hal-02423695}

Submitted on 27 Mar 2020

HAL is a multi-disciplinary open access archive for the deposit and dissemination of scientific research documents, whether they are published or not. The documents may come from teaching and research institutions in France or abroad, or from public or private research centers.
L'archive ouverte pluridisciplinaire HAL, est destinée au dépôt et à la diffusion de documents scientifiques de niveau recherche, publiés ou non, émanant des établissements d'enseignement et de recherche français ou étrangers, des laboratoires publics ou privés. 


\title{
On Limitations of Using Silicon Photo-Multipliers for Underwater Wireless Optical Communications
}

\author{
Tasnim Hamza, Mohammad Ali Khalighi \\ Aix-Marseille University, CNRS, Centrale Marseille, Institut Fresnel, Marseille, France \\ (Email: Ali.Khalighi@fresnel.fr)
}

\begin{abstract}
This paper considers the use of Silicon photomultipliers in underwater wireless optical communication systems. We study the limitations arising from the limited bandwidth of these components as well as the solar background noise in relatively shallow waters. In particular, we show the interest of frequency-domain equalization in increasing the link data rate using on-off-keying and pulse-position modulation. Also, for a worst-case scenario, we determine the operation depth (with respect to the receiver) beyond which the link performance is not affected by the solar noise.
\end{abstract}

\section{INTRODUCTION}

Underwater wireless optical communication (UWOC) is a promising technology that enables energy-efficient highspeed data transmission with reduced environmental impact, as compared with the traditional acoustic-based communications [1]-[4]. Recently, Silicon Photo-multipliers (SiPMs) have received particular attention for use in UWOC systems thanks to their high sensitivity and gain as well as considerably lower implementation complexity, as compared with photo-multiplier tubes (PMTs) [5].

One drawback of SiPMs is the non-linear distortion on the received signal due to the limited dynamic range (DR) of these components. This specially impacts the system operability at relatively short ranges [5]. Also, the limited bandwidth (BW) of SiPMs imposes restrictions on the maximum transmission rate of the system, which may be further aggravated by the relatively low modulation $\mathrm{BW}$ of the high-power LEDs usually used at the transmitter (Tx). On the other hand, the use of these highly sensitive detectors imposes limitations on link deployment at relatively shallow waters where the solar background noise could severely affect the link performance.

Our aim in this paper is to investigate the performance improvement offered by equalization at the receiver $(\mathrm{Rx})$ in terms of the maximum achievable link data rate, conditioned to a specific requirement on the bit-error-rate (BER). For this, we consider transmission schemes based on intensity modulation at the Tx and direct detection at the $\mathrm{Rx}$, namely using on-off keying (OOK) and pulse position modulation (PPM), which are amongst relevant modulation techniques in this context [6]. Furthermore, we study the effect of solar noise on the Rx performance and determine the limitations on the operation depth of an SiPM-based UWOC link.

The remainder of this paper is organized as follows: After describing our main assumptions regarding the Tx, the $\mathrm{Rx}$, and the aquatic channel in Section II, we review in Section III the mathematical modeling of an SiPMbased Rx. Then, the equalization of the aggregate channel is addressed in Section IV and the resulting performance improvement is discussed in Section V. Lastly, Section VII concludes this work.

\section{MAIN ASSUMPTIONS AND SYSTEM MODEL}

We consider a point-to-point communication link, as depicted in Fig. 1 with an LED-based Tx. We consider the SiPM output as discrete, consisting in photon count values. We model the LED radiation optical power $P_{\mathrm{Tx}}$ by a generalized Lambertian model with azimuthal symmetry as follows:

$$
P_{\mathrm{Tx}}=P_{\mathrm{t}} \frac{m+1}{2 \pi} \cos ^{m}(\phi) \quad[\mathrm{W} / \mathrm{sr}],
$$

where $P_{\mathrm{t}}$ is the total emitted power at the Tx, $\phi \in[0, \pi / 2]$ denotes the angle of irradiance, and $m$ stands for the Lambertian order, given by $m=-\ln (2) / \ln \left(\cos \left(\phi_{1 / 2}\right)\right)$, with $\phi_{1 / 2}$ being the Tx semi-angle at half-power. We model the optical power loss by an exponential decay model, which is suitable for the case of using an LED at the Tx, see [7]-[10]. This way, assuming perfect Tx-Rx alignment and negligible turbulence effect, the captured power $P_{\mathrm{Rx}}$ at the $\mathrm{Rx}$ is given by:

$$
P_{\mathrm{Rx}}=P_{\mathrm{Tx}} \exp (-K Z) \frac{A}{Z^{2}},
$$

where $Z$ is the link distance, $A$ is the photo-sensitive area of the SiPM, and $K$ denotes the diffuse attenuation coefficient [10]. We further neglect the effect of pulse broadening (i.e. channel frequency selectivity) due to light scattering in water, which is valid for low-to-medium turbidity waters [4], [11]-[13]

\section{SiPM MODELING AND PARAMETERS}

When the incoming light (and thus the photon flux) hits the photo-sensitive area of the SiPM, each underlying SPAD (Single-Photon Avalanche Diode, also called micro-cell) proceeds to individual photon counting. Given that it is biased in the Geiger mode, the SPAD can be assumed as an ideal photon counter whose detection process can be approximated by a Poisson distribution, representing the shot noise effect [14]. The SPAD generates an output pulse as a reaction to the detection of a photon. Counting these pulses over a time interval $T$ is equivalent to counting the number of detected photons over $T$; The sum of individual 


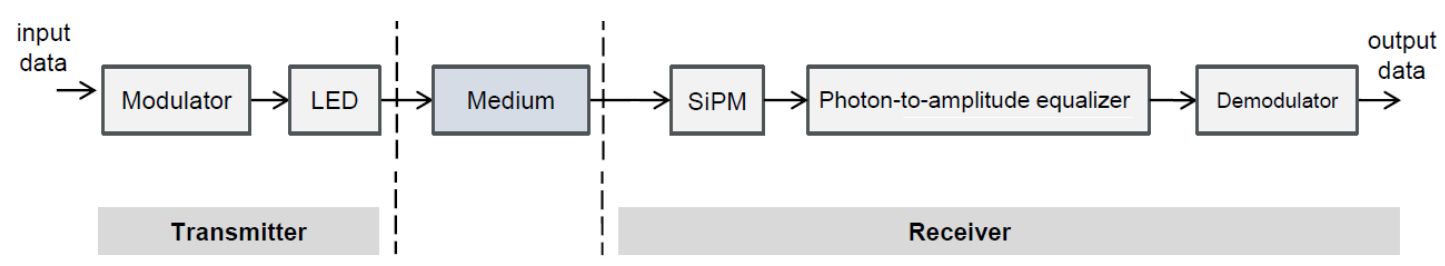

Fig. 1: Block diagram of the UWOC link using an SiPM at the Rx.

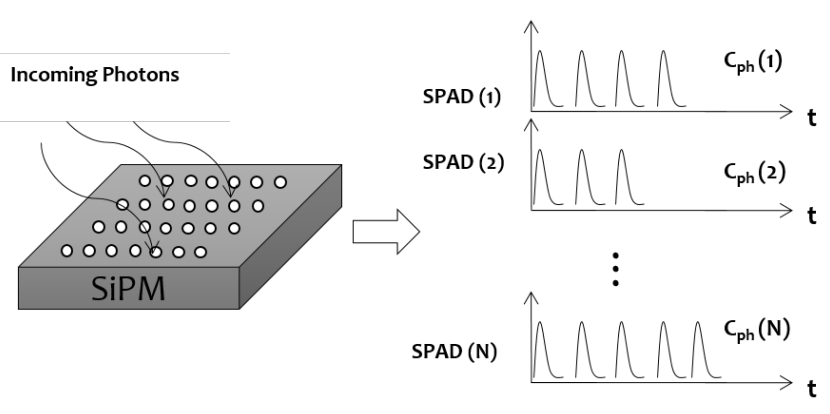

Fig. 2: Photon-counting procedure with an SiPM PD

SPAD counts forms the SiPM output [14]. Thus, the information about the detected light intensity is generated by the internal superposition of the signals of all fired microcells [15]. This is schematically illustrated in Fig. 2, where $N$ stands for the number of SPADs composing the SiPM and $C_{\mathrm{ph}}(i)$ corresponds to the photon count at a given time related to SPAD number $i$ (taking values of 0 or 1 ). We denote by $C_{\mathrm{ph}}$ the SiPM photon-count output. We have:

$$
C_{\mathrm{ph}}=\sum_{i=1}^{N} C_{\mathrm{ph}}(i)
$$

It should be noted that this operation procedure is only valid when the SiPM is operating in the linear mode, i.e. where its output is proportional to the input signal magnitude. We model the SiPM photo-detection by a Poisson distribution [14] as follows:

$$
\operatorname{Pr}\left(C_{\mathrm{ph}}=k\right)=\exp (-\mu) \frac{\mu^{k}}{k !} .
$$

Here, $\mu$ denotes the average photon count and is expressed as a function of $P_{\mathrm{Rx}}$ as follows [16]:

$$
\mu=\left(\frac{\Upsilon_{\mathrm{PDE}} P_{\mathrm{Rx}}}{E_{\mathrm{ph}}}+f_{\mathrm{DCR}}\right)\left(1+P_{\mathrm{AP}}+P_{\mathrm{CT}}\right) T,
$$

where $T$ denotes the bit duration and $E_{\mathrm{ph}}=\hbar c / \lambda$ is the energy of photon, with $\hbar, c$, and $\lambda$ being the Planck constant, the speed of light, and the light wavelength, respectively. Also, $\Upsilon_{\mathrm{PDE}}, f_{\mathrm{DCR}}$, and $P_{\mathrm{AP}}$ and $P_{\mathrm{CT}}$ stand for the SiPM photon detection efficiency, dark count rate, and after-pulsing and cross-talk probabilities, respectively. Another key parameter of an SiPM is its dead-time, that we denote by $\tau_{\mathrm{d}}$, which is the time needed by a micro-cell to recharge after being triggered by an incoming photon and during which the SPAD is unable to detect any new arriving photon.
TABLE I: SiPM parameters.

\begin{tabular}{|l|l|}
\hline Parameter & Value \\
\hline \hline SiPM PDE, $\Upsilon_{\mathrm{PDE}}$ & 0.24 \\
SiPM area & $9 \mathrm{~mm}^{2}$ \\
SiPM Dark Count Rate, $f_{\mathrm{DCR}}$ & $6.6 \mathrm{MHz}$ \\
SPAD dead-time, $\tau_{\mathrm{d}}$ & $100 \mathrm{~ns}$ \\
Number of SPAD in SiPM, $N_{\mathrm{SPAD}}$ & 10998 \\
Probability of Cross-Talk, $P_{\mathrm{CT}}$ & $0.03 \%$ \\
Probability of After-Pulsing, $P_{\mathrm{AP}}$ & $0.2 \%$ \\
\hline
\end{tabular}

There are two types of quenching mechanisms used to handle the SPAD during the breakdown: passive and active quenching, see [5], [17], [18]. In contrast to the average measured photon count $\mu$ given in (4), we define $\mu_{\mathrm{PQ}}$ and $\mu_{\mathrm{AQ}}$ respectively as the real average photoncount of a passively-quenched (PQ)- and actively-quenched (AQ)- SiPM during a bit time $T$. The relation between $\mu_{\mathrm{PQ}}$ and $\mu$, for instance, can be formulated based on a paralyzable-detector model adopted from nuclear particle physics instruments [14], [19], as follows:

$$
\begin{aligned}
& \mu_{\mathrm{PQ}}=\mu \exp \left(-\frac{\mu \tau_{\mathrm{d}}}{T N}\right) \\
& \mu_{\mathrm{AQ}}=\frac{\mu}{1+\mu \tau_{\mathrm{d}} /(T N)} .
\end{aligned}
$$

In order to illustrate these relationships, inspired by a similar study in [14], we have simulated the output photon count of an SiPM versus the input optical power $P_{\mathrm{Rx}}$, for the two counting time intervals of $T=0.1$ and $1 \mu \mathrm{s}$, assuming $N=10998$ and $\tau_{d}=100 \mathrm{~ns}$. The other considered SiPM parameters are presented in Table I, which correspond to the SensL B-series 30020 component [20] for the PQSiPM. The results are presented in Fig. 3, which clearly shows the two operation regimes of the SiPM. During the first regime, the curve of the output photon count versus $P_{\mathrm{Rx}}$ exhibits a linear behavior until a maximum photon count (depending on $T$ ). Above this limit, the photon count is constant for the case of AQ-SiPM whereas it drops sharply and marks the start of the saturation regime for the case of PQ-SiPM. Note that the saturation point in terms of the maximum accepted photon rate (or equivalently $\left.P_{\mathrm{Rx}}\right)$ is constant for both counting time intervals, and is on the order of $10^{12}$ photons/s. This confirms the fact that, considering the dead-time constraint, an SPAD is practically able to detect photons at a maximum rate of $\approx 1 / \tau_{d}$ [19], [21], resulting in a maximum rate of $\approx N / \tau_{d}$ for the SiPM, here about $10^{12}$ photons/s. In the sequel, we consider only the case of PQ-SiPM, since relatively large DR components are currently available on the market. 


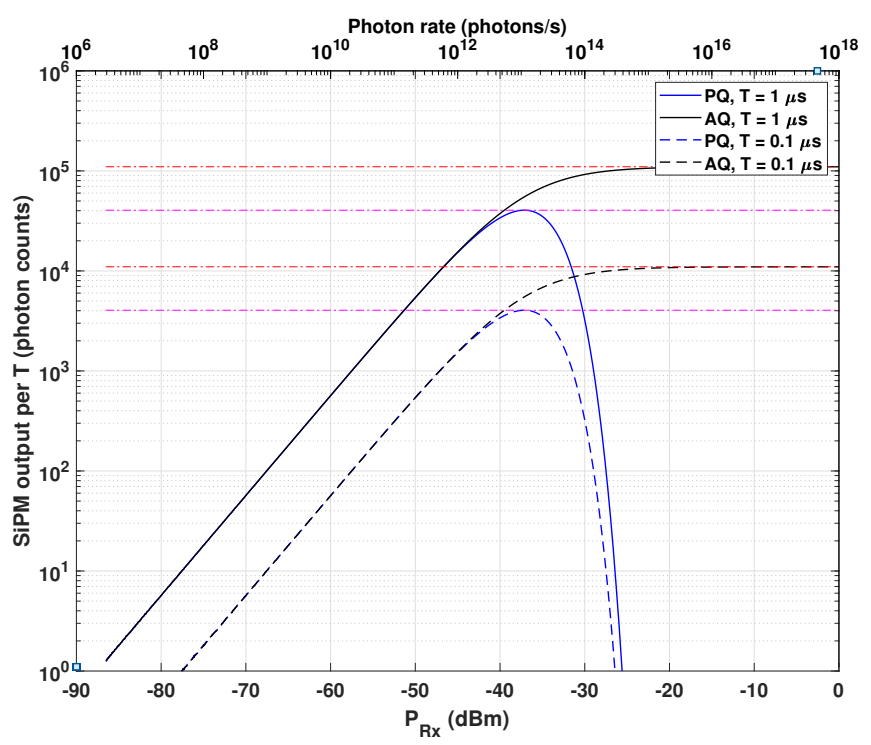

Fig. 3: AQ and PQ SiPM photon-count output as a function of optical received power $P_{\mathrm{Rx}}$ and the incoming photon flux.

\section{Equalization of the Aggregate Channel}

Figure 4 shows the block diagram of the Tx and the $\mathrm{Rx}$ of an UWOC link using frequency-domain equalization (FDE). Information bits to be transmitted are first mapped according the modulation scheme. They are next grouped by blocks of length $N_{\text {fft }}$ which is the FFT size. Then, a cyclic prefix (CP) of length $M$ is introduced at the beginning of each block, which consists of a duplicate of the $M$ last symbols of each block. The CP forms a guard interval between the successive blocks in the purpose of preventing inter-block interference. Furthermore, it allows circular convolution, as the block of symbols may be regarded as periodic with period $N_{\mathrm{fft}}$ [22]. After pulse shaping, parallel to serial (P/S) and digital to analog (D/A) conversion, the transmitted signal can be written as follows:

$$
s(t)=\sum_{i=-\infty}^{\infty} a(i) g(t-i T) * h_{\mathrm{LED}}(t)
$$

where $a(i)$ denotes the transmitted optical power level corresponding to the $i^{\text {th }}$ symbol (or time slot), and $g(t)$ the pulse shaping filter impulse response. Here we consider $g(t)$ as having a rectangular shape, for the sake of simplicity.

At the Rx side, after photo-detection and prior to timesampling (here it is implicit given that the SiPM output is considered to be in discrete time), the signal can be written as:

$$
\begin{aligned}
r(t) & =\sum_{i=-\infty}^{\infty} b(i) g(t-i T) * h_{\mathrm{LED}}(t) * h_{\mathrm{ch}}(t) * h_{\mathrm{SiPM}}(t) \\
& =\sum_{i=-\infty}^{\infty} b(i) g(t-i T) * h_{\mathrm{eq}}(t) .
\end{aligned}
$$

Here, $b(i)$ denotes the number of photons counted by SiPM, corresponding to $a(i)$, and includes the cross-talk and afterpulse components, see Section III. Also, $h_{\mathrm{LED}}(t), h_{\mathrm{ch}}(t)$, and $h_{\mathrm{SiPM}}(t)$ represent the impulse responses of the LED, the aquatic channel, and the SiPM, respectively. We will refer to $h_{\mathrm{eq}}(t)$ as the aggregate channel impulse response, which includes the effect of the aquatic channel and the BW-limited LED and SiPM.

After removing the $\mathrm{CP}$, equalization of the aggregate channel is performed in the frequency domain. Here we consider zero-forcing (ZF) based equalization, which has the advantage of implementation simplicity. Notice that, for larger signal BWs, a larger FFT size should be used in order to satisfy the condition of almost flat sub-channels. Also, the CP length should be set carefully, depending on the aggregate channel delay spread and the signal BW [23].

\section{BER PERFORMANCE IMPROVEMENT BY FDE}

In the simulation results that we present here, the LED is considered to emit at $\lambda=470 \mathrm{~nm}$ (where the considered SiPM exhibits its maximum responsivity [24]), with a Lambertian order of $m=45$, corresponding to $\phi_{1 / 2} \approx 10^{\circ}$. Also, we conduct our study for the case of clear ocean waters, with a typical Chlorophyll concentration of $C=$ $0.5 \mathrm{mg} / \mathrm{m}^{3}$ corresponding to a diffuse attenuation coefficient of $K=0.08 \mathrm{~m}^{-1}$, according to the Woźniak's bio-optical model [25] (see more explanation in the Appendix). We set $N_{\mathrm{fft}}=256$ and fix CP appropriately, depending on the signal BW. The LED and SiPM impulse responses are modeled by a first-order low-pass filter with 3-dB cut-off frequencies of 2 and $10 \mathrm{MHz}$, respectively, see [5].

To illustrate the interest of employing FDE in the considered UWOC system, we fix the link range $Z$ and study the BER performance as a function the data rate. Two modulation schemes of OOK and $L$-PPM are considered. Let us denote by $P_{\mathrm{OOK}, \mathrm{On}}$ and $P_{\mathrm{OOK}, \mathrm{Off}}$ the transmitted intensity levels $P_{t}$ for OOK modulation, corresponding to On and Off symbols, respectively. Similarly, we define $P_{\text {PPM,On }}$ and $P_{\text {PPM,Off }}$ as the intensity levels corresponding to On and Off time slots in a PPM symbol, respectively. For OOK, we set $P_{\mathrm{OOK}, \mathrm{On}}=1$ and consider an extinction ratio of $\mathrm{EXT}=P_{\mathrm{OOK}, \mathrm{On}} / P_{\mathrm{OOK}, \mathrm{Off}}=0.2$, to allow fast switching of the LED. For the case of PPM, in order to make a fair comparison with the OOK case, we fix the average transmit optical power per symbol duration. There, we have to relax the constraint on EXT but we fix the same intensity for Off state, i.e., we set $P_{\mathrm{PPM}, \text { Off }}=P_{\mathrm{OOK} \text {,Off. }}$. To have the same average transmit optical power, it can be easily shown that, we should set $P_{\mathrm{PPM}, \mathrm{On}}=\frac{L}{2} P_{\mathrm{OOK}, \mathrm{On}}-\frac{L-2}{2} P_{\mathrm{OOK} \text {,Off }}$.

We have presented the simulation results for the case of OOK and two link distances of $Z=30$ and $40 \mathrm{~m}$ in Fig. 5 . We notice a significant performance improvement with channel equalization in terms of the maximum achievable data rate at a given target BER. This is in particular the case for $Z=30 \mathrm{~m}$, where the link performance is obviously limited by the inter-symbol interference (ISI) arising from BW limitation of the aggregate channel. For $Z=40 \mathrm{~m}$ where the received signal intensity is lower, we still notice an interesting performance improvement with FDE but here the link performance is likely limited by the relatively low signal-to-noise ratio (SNR) at the Rx. For instance, for a target BER of $10^{-4}$, the increase in the maximum achievable data rate is around 22.5 and $5 \mathrm{Mbps}$ for $Z=30$ and $40 \mathrm{~m}$, respectively. We see that channel equalization 
(a)

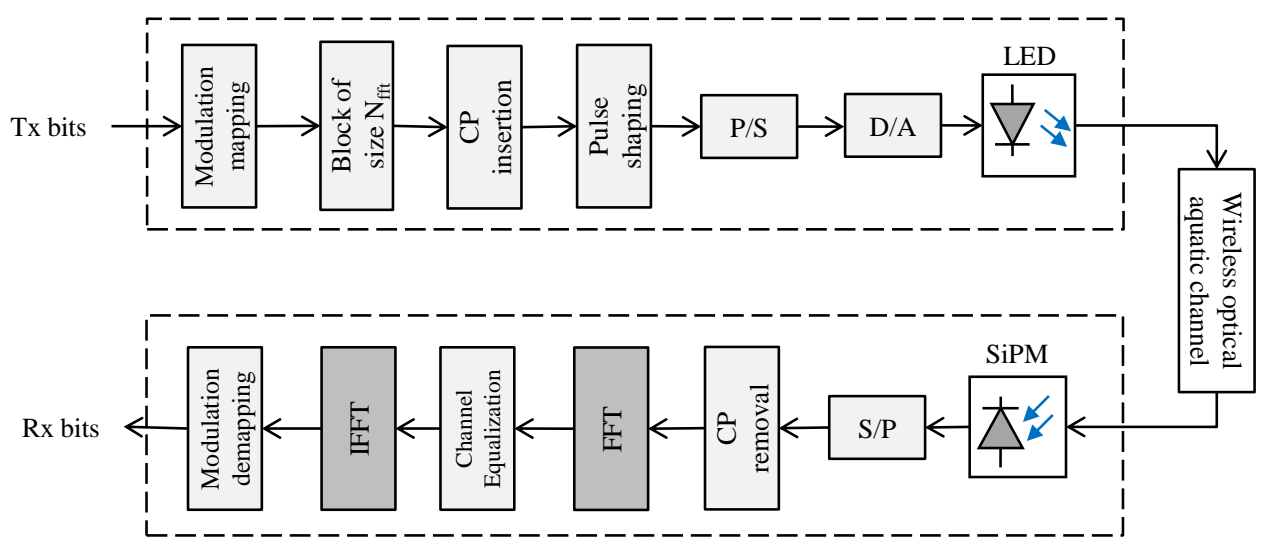

(b)

Fig. 4: Block diagram of an UWOC (a) Tx and (b) Rx for an FDE-based transmission.

allows increasing the transmission rate quite beyond the limitation imposed by LED and SiPM BWs.

For the PPM case, we have shown the BER plots with and without FDE at the Rx in Figs. 6 and 7 for $Z=30$ and $40 \mathrm{~m}$, respectively. Note that $R_{b}$ still refers to the bit rate; the symbol and slot durations here correspond to $R_{b} / \log _{2} L$ and $L R_{b} / \log _{2} L$. A larger $L$ (i.e., a higher order modulation) results in a shorter slot duration and an increased inter-slot interference and ISI. So, we normally expect a better benefit of FDE for higher $L$. This is confirmed in the presented results where we see a more considerable performance degradation by increasing $L$ when no equalization is done at the Rx. From Fig. 6, for $Z=40 \mathrm{~m}$ and with FDE, the increase in the maximum achievable bitrate at a target BER of $10^{-4}$ is about $10.5,13$, and $15 \mathrm{Mbps}$ for the cases of 4-, 8-, and 16-PPM, respectively. This increase is more significant for $Z=30 \mathrm{~m}$ (as we noticed from Fig. 5 for OOK modulation) and is about 18.5, 25, and $28 \mathrm{Mbps}$ for $L=4,8$, and 16, respectively, see Fig. 7 .

Lastly, note that here we considered the case of uncoded modulation. Obviously, the BER can further be significantly reduced by using channel coding, see [26], [27] for instance.

\section{BACKGROUnd Solar NoISE EFFECT}

Let us now study the limitation in terms of operation depth on the use of SiPMs when the Rx is subject to the solar background noise. Similar to a recent work [10] where we investigated the impact of solar noise on the link performance for PIN, APD and PMT photo-detectors, here we study the link BER variation versus the $\mathrm{Rx}$ operation depth $D$. We consider a worst case scenario where the sun is at the zenith, and the Rx plan is directed towards the sea surface (without any obstacle), hence capturing the maximum solar irradiation. Although this worst case is not likely to happen in practice, the interest of considering it is to determine the maximum limitation on the link operation depth. In other words, our aim is to determine the pessimistic minimum depth, where the link performance is not affected by the solar noise. Obviously, for a ultrasensitive component like an SiPM, the impact of solar noise

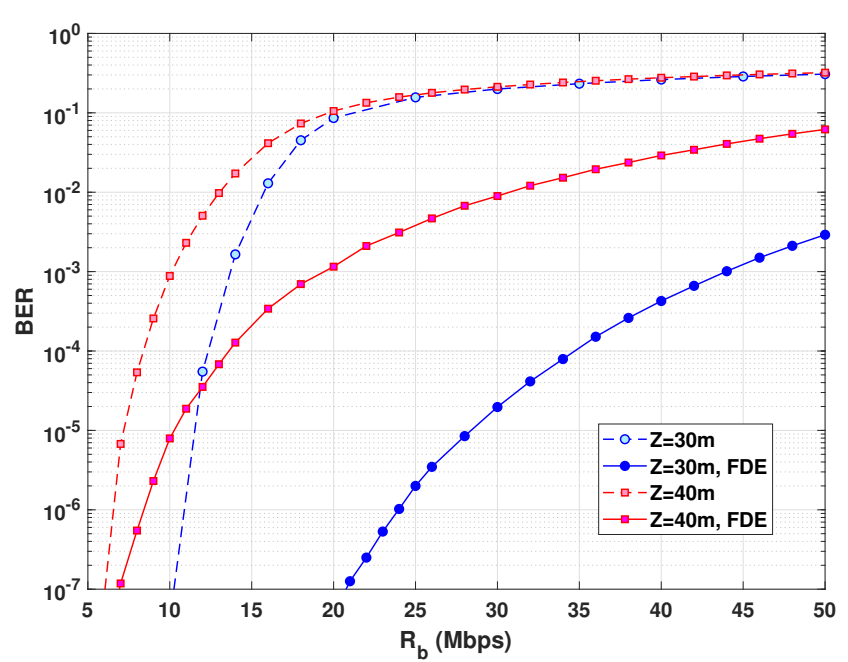

Fig. 5: BER performance as a function of bit rate with and without FDE, OOK modulation, PQ-SiPM-based Rx,

$$
Z=30,40 \mathrm{~m} \text {. }
$$

at low depths is much more important than in the case of a PIN or an APD [10].

To study the effect of solar noise with respect to the Rx operation depth, we fix the link span to $Z=69 \mathrm{~m}$, which corresponds to a target BER of around $10^{-4}$ for OOK modulation with EXT $=0$ and in the absence of solar noise. The solar irradiance is set to $1.8 \mathrm{~W} \mathrm{~m}^{-2} \mathrm{~nm}^{-1}$ [28]. Then, we obtain through simulations the BER for different depths $D$, as presented in Fig. 8. We notice that for relatively low depths, i.e., $D<200 \mathrm{~m}$, the link performance is highly affected by the solar ambient light, resulting in a BER higher that $10^{-2}$. The BER decreases with increase in $D$ due to a lower background noise level captured at the $\mathrm{Rx}$, until it reaches the background-noise-free performance, around $10^{-4}$ for $D>250 \mathrm{~m}$. As expected, in the presence of solar noise, the use of a high sensitivity SiPM in relatively shallow waters is considerably more restrictive, compared to a PIN- or an APD-based Rx [10]. 


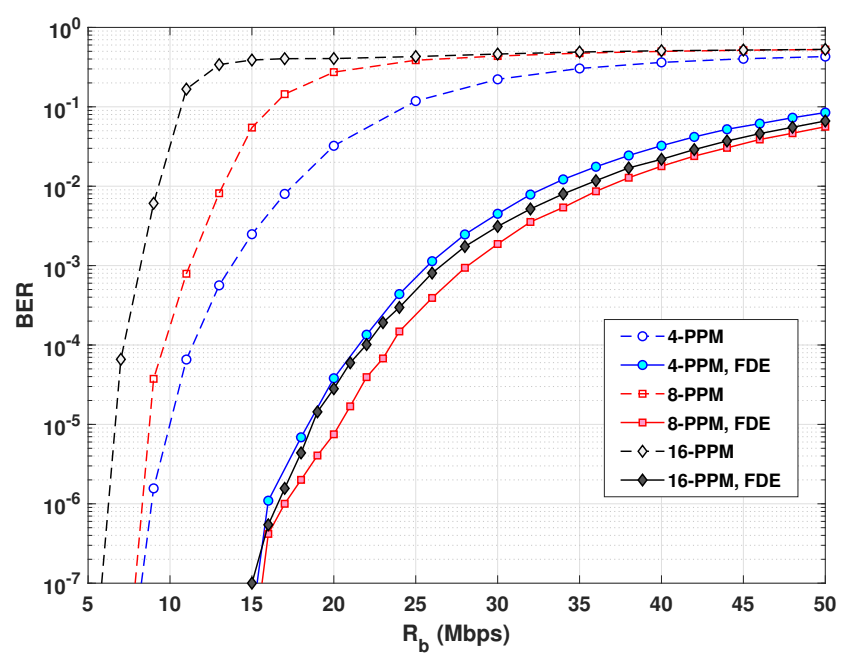

Fig. 6: BER performance as a function of bit rate with and without FDE at the Rx, PPM modulation, PQ-SiPM-based Rx, $Z=40 \mathrm{~m}$.

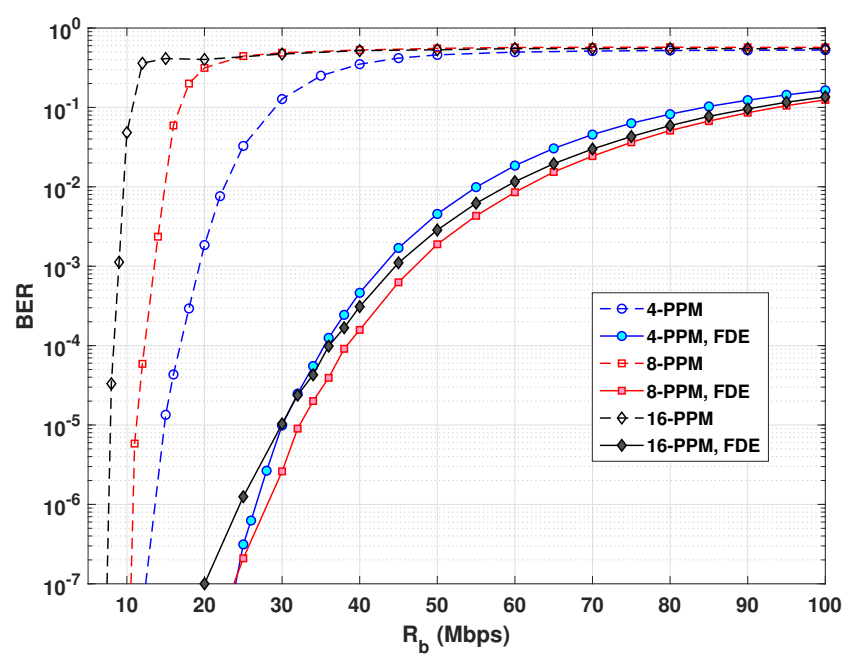

Fig. 7: BER performance as a function of bit rate with and without FDE at the Rx, PPM modulation, PQ-SiPM-based Rx, $Z=30 \mathrm{~m}$.

\section{CONCLUSIONS}

In this paper, we considered the use of SiPMs in UWOC links and discussed the limitations on the data rate arising from the $\mathrm{Tx} / \mathrm{Rx}$ components. We studied the performance improvement in terms of maximum achievable data rate through channel equalization at the Rx. FDE was considered as an appropriate solution with reasonable computational complexity, as compared with time-domain equalization. We showed that, this way we can benefit from a substantial improvement in the link performance at the same time of a high sensitivity offered by the SiPM. We also investigated the limitations in terms of minimum $\mathrm{Rx}$ operation depth in the presence of solar background noise and determined the depth limit beyond which the link performance is not affected by the solar noise.

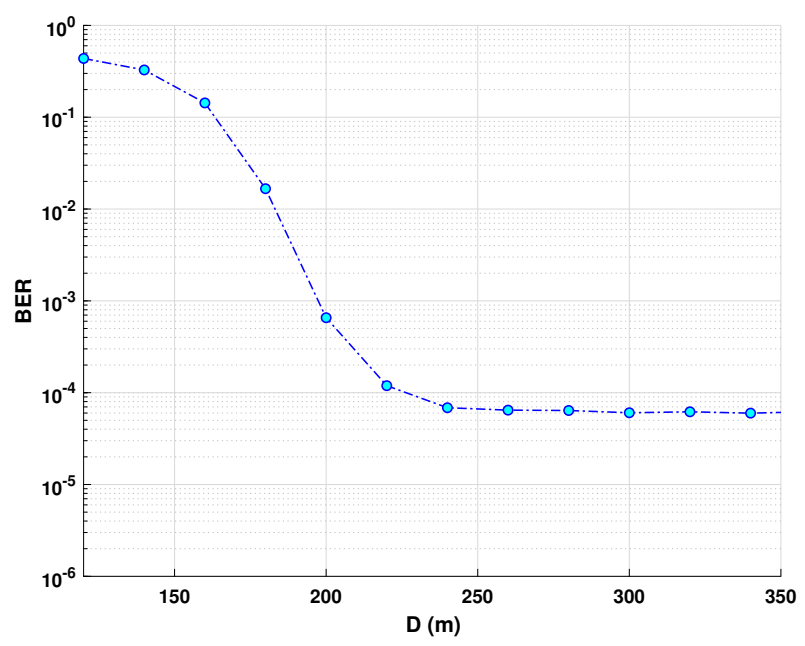

Fig. 8: Impact of solar noise on the BER performance of an SiPM-based system, OOK modulation with $\mathrm{EXT}=0, Z=69 \mathrm{~m}$

\section{ACKNOWLEDGMENT}

This work was supported in part by the French PACA Regional Council and the French Research Institute for Exploitation of the Sea (IFREMER). The authors would like to thank Mr. Pierre Léon and Dr. Jan Opderbecke from IFREMER, La-Seyne-sur-Mer, for the fruitful discussions.

\section{APPENDIX}

We provide here a brief explanation on the Woźniak's bio-otical model. This water classification model is based on the statistical analysis of experimental data collected in different waters (lakes, seas, and oceans), with different Chlorophyll concentrations $C$. It is very similar to a formerly-presented model, i.e., that of Smith and Baker [25], and provides an analytic relationship between the diffuse attenuation coefficient $K$ and $C$. According to this model, we have:

$$
K(\lambda)=K_{\mathrm{w}}(\lambda)+C\left[C_{1}(\lambda) \exp \left(-a_{1}(\lambda) C\right)+k_{\mathrm{c}, \mathrm{n}}(\lambda)\right]
$$

where $K_{\mathrm{w}}(\lambda)$ denotes the downward irradiance attenuation due to pure water, and $C_{1}(\lambda)$ and $a_{1}(\lambda)$ are statistically derived parameters, whose values and the description of their computation procedure are presented in [25]. Also, $k_{\mathrm{c}, \mathrm{n}}(\lambda)$ is the light attenuation factor with $n=1$ and 2 corresponding to low and high Chlorophyll concentrations, respectively. According to [25], this analytical expression matches several experimental measurements, for instance, for $\lambda=440 \mathrm{~nm}$ and $C$ varying from 0.01 to $100 \mathrm{mg} / \mathrm{m}^{3}$.

\section{REFERENCES}

[1] M. A. Khalighi, C. J. Gabriel, L. M. Pessoa, and B. Silva, Visible Light Communications: Theory and Applications. CRC-Press, 2017, ch. Underwater Visible Light Communications, Channel Modeling and System Design, pp. 337-372.

[2] F. Hanson and S. Radic, "High bandwidth underwater optical communication," Applied Optics, vol. 47, no. 2, pp. 277-283, Jan. 2008 
[3] M. A. Khalighi, C. Gabriel, T. Hamza, S. Bourennane, P. Lon, and V. Rigaud, "Underwater wireless optical communication; recen advances and remaining challenges," IEEE International Conference on Transparent Optical Networks (ICTON), pp. 1-4, July 2014 invited paper, Graz, Austria.

[4] B. Cochenour, L. Mullen, and J. Muth, "Temporal response of the underwater optical channel for high-bandwidth wireless laser communications," IEEE Journal of Oceanic Engineering, vol. 38, no. 4, pp. 730-742, Oct. 2013

[5] M. A. Khalighi, T. Hamza, S. Bourennane, P. Léon, and J. Opderbecke, "Underwater wireless optical communications using silicon photo-multipliers," IEEE Photonics Journal, vol. 9, no. 4, 2017, dOI 10.1109/JPHOT.2017.2726565.

[6] C. Gabriel, M. A. Khalighi, S. Bourennane, P. Léon, and V. Rigaud, "Investigation of suitable modulation techniques for underwater wireless optical communication," Int. Workshop Optical Wireles Commun. (IWOW), pp. 1-3, Oct. 2012, Pisa, Italy.

[7] S. Q. Duntley, "Light in the sea," Journal of the Optical Society of America (JOSA), vol. 53, no. 2, pp. 214-233, Feb. 1963.

[8] C. Mobley, Light and Water: Radiative Transfer in Natural Waters. Academic Press, 1994.

[9] J. W. Giles and I. N. Bankman, "Underwater optical communication systems. part 2 : Basic design considerations," Military Communications Conference (MILCOM), vol. 3, pp. 1700-1705, Oct. 2005 , Atlantic City, NJ, USA

[10] T. Hamza, M. A. Khalighi, S. Bourennane, P. Léon, and J. Opderbecke, "Investigation of solar noise impact on the performance of underwater wireless optical communication links," Optics Express, vol. 24, no. 22, pp. 25 832-25845, Oct. 2016.

[11] B. M. Cochenour, L. J. Mullen, and A. E. Laux, "Characterization of the beam-spread function for underwater wireless optical communications links," IEEE Journal of Oceanic Engineering, vol. 33 , no. 4, pp. 513-521, Oct 2008.

[12] C. Gabriel, M. A. Khalighi, S. Bourennane, P. Léon, and V. Rigaud, "Channel modeling for underwater optical communication," IEEE Workshop on Optical Wireless Communications, Global Communication Conference, pp. 833-837, Dec. 2011, Houston, TX.

[13] _ - "Monte-carlo-based channel characterization for underwater optical communication systems," IEEE/OSA J. Opt. Commun. Networking, vol. 5, no. 1, pp. 1-12, Jan. 2013.

[14] Y. Li, M. Safari, R. Henderson, and H. Haas, "Nonlinear distortion in SPAD-based optical OFDM systems," Global Communications Conference (GLOBECOM), Workshop on Optical Wireless Communications, Dec. 2015, San Diego, CA.

[15] Kektek, "Products SiPM Technology Working Principle," http://www.ketek.net/, 2016.

[16] Y. Li, S. Videv, M. Abdallah, K. Qaraqe, M. Uysal, and H. Haas,
"Single photon avalanche diode (SPAD) VLC system and application to downhole monitoring," in Global Communications Conference (GLOBECOM), 2014 IEEE, Dec. 2014, pp. 2108-2113.

[17] N. Otte, "The Silicon Photomultiplier - a new device for high energy physics, astroparticle physics, industrial and medical applications,' International Symposium on Detectors for Particle, Astroparticle and Synchrotron Radiation Experiments (SNIC), pp. 1-9, Apr. 2006, Stanford, California.

[18] Y. Li, M. Safari, R. Henderson, and H. Haas, "Optical OFDM with single-photon avalanche diode," Photonics Technology Letters, IEEE, vol. 27, no. 9, pp. 943-946, May 2015.

[19] A. Eisele, R. Henderson, B. Schmidtke, T. Funk, L. Grant, J. Richardson, and W. Freude, "185 MHz count rate, $139 \mathrm{~dB}$ dynamic range single-photon avalanche diode with active quenching circuit in 130nm CMOS technology," International Image Sensor Workshop, pp. 278-281, June 2011, Hokkaido, Japan.

[20] SensL, "B-Series Fast, Blue-Sensitive Silicon Photomultiplier Sensors Datasheet," http://www.sensl.com/, 2013.

[21] S. Donati and T. Tambosso, "Single-photon detectors: From traditional PMT to solid-state SPAD-based technology," IEEE Journal of Selected Topics in Quantum Electronics, vol. 20, no. 6, pp. 204-211, Nov. 2014.

[22] M. Wolf, S. A. Cheema, and M. Haardt, Optical Wireless Communications, an Elerging Technology. Springer, 2016, ch. Block Transmission with Frequency Domain Equalization for VLC, pp. 299-323.

[23] M. A. Khalighi, S. Long, S. Bourennane, and Z. Ghassemlooy, "PAM and CAP-based transmission schemes for visible-light communications," IEEE Access Journal, Special issue on Optical Wireless Technologies for 5G Communications and Beyond, vol. 5, pp. 27002 27013 , Oct. 2017.

[24] SensL, "Introduction to the Silicon Photomultiplier, Technical Note," http://www.sensl.com/, 2011.

[25] B. Woźniak and V. N. Pelevin, "Optical classifications of the seas in relation to phytoplankton characteristics," Oceanologia, vol. 31, pp. 25-55, Dec. 1991.

[26] F. Mattoussi, M. Khalighi, and S. Bourennane, "Improving the performance of underwater wireless optical communication links by channel coding," Applied Optics, vol. 57, no. 9, pp. 2115-2120, Mar. 2018

[27] F. Xu, M. A. Khalighi, and S. Bourennane, "Coded PPM and multipulse PPM and iterative detection for Free-Space optical links,' IEEE/OSA Journal of Optical Communications and Networking, vol. 1, no. 5, pp. 404-415, Oct. 2009.

[28] E. B. C. Mobley and C. Roesler, Ocean Optics Web Book, http://www.oceanopticsbook.info/, 2016. 